\title{
Evaluation of $\mathrm{His}_{6}$-metal assemblies as a drug delivery vehicle in the treatment of anterior segment disease using a corneal inflammation model
}

Liwen Wang, a Shan Luo, , Hongyan Xu, , Xiaoxiao Wu, , Pengyan Hao, a Yagang Zhang, ${ }^{c}$ Wenjuan Huang, ${ }^{a,{ }^{*}}$ Xingjie Zan ${ }^{a, b,{ }^{*}}$

a. School of Ophthalmology and Optometry, Eye Hospital, School of Biomedical Engineering, Wenzhou Medical University, Wenzhou, Zhejiang Province, 325035, PR China

b. Wenzhou Institute of Biomaterials and Engineering, CNITECH, Chinese Academy of Sciences, Wenzhou, Zhejiang Province, 325011, PR China

c. School of Materials and Energy, University of Electronic Science and Technology of China, Chengdu 611731, PR China ${ }^{*}$ Corresponding author: Xingjie Zan, xjzan2000@hotmail.com 

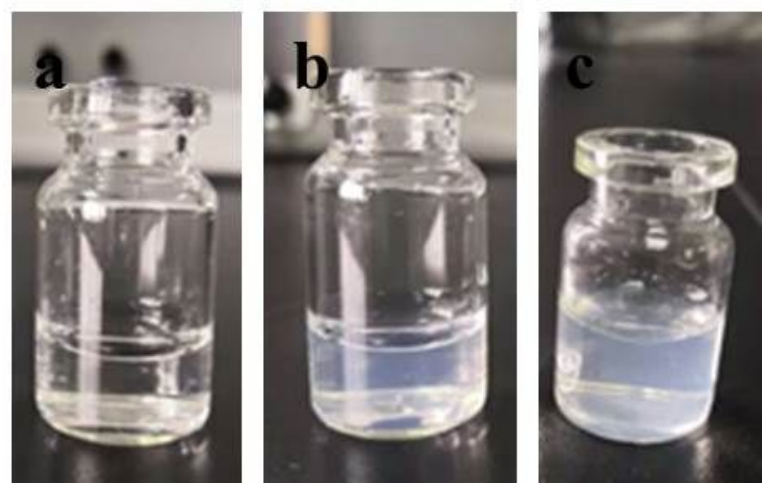

Figure S1. Photo images of (a) $\mathrm{His}_{6}$ or mixture of $\mathrm{His}_{6}$ and Dexp at $\mathrm{pH} 8$ before zion adding, (b) generated HmA and(c) generated Dexp@HmA. 


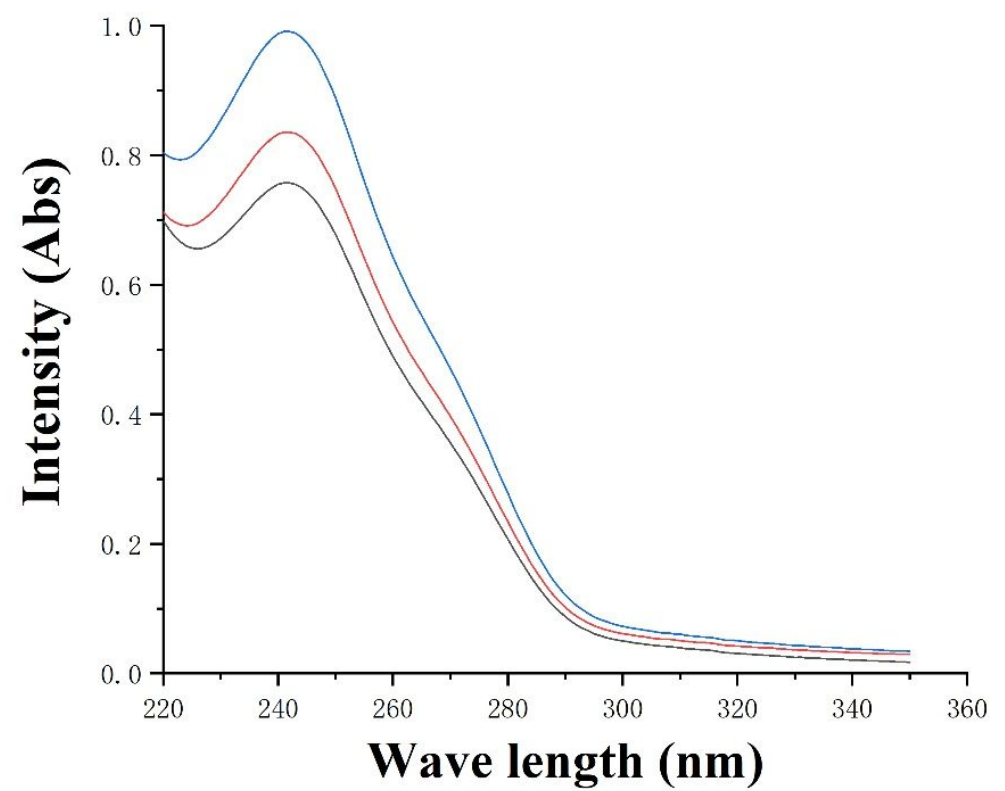

Figure S2. UV-Vis spectra of the original free Dexp (black) before encapsulation, Dexp (blue) in Dexp@HmA, and Dexp (red) after released from Dexp@HmA. 


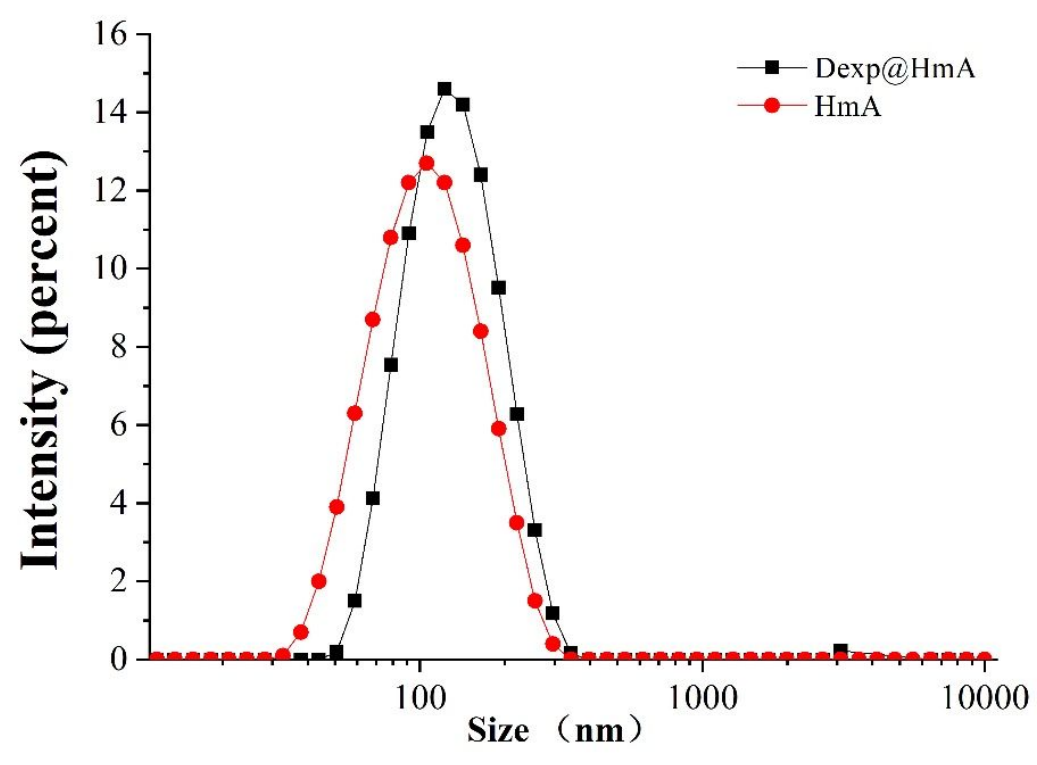

Figure S3. Size distribution of HmA and Dexp@HmA. 

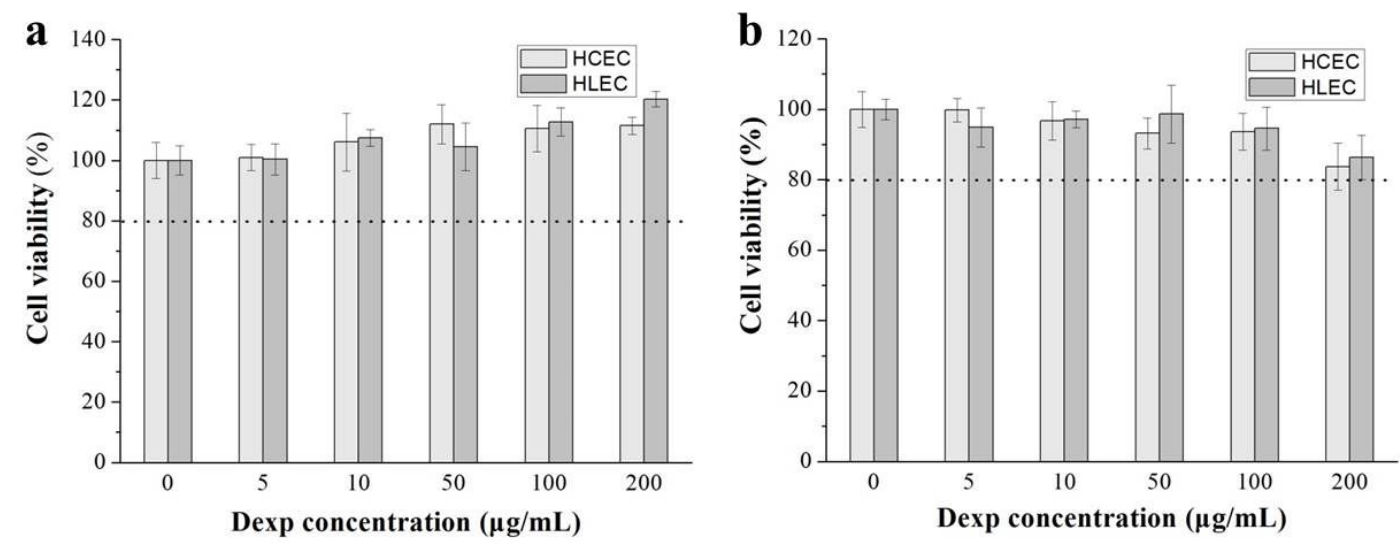

Figure S4. Cell viability of HCEC and HLEC incubated with different concentrations of Dexp for (a) 24h and (b) $48 \mathrm{~h}$. 

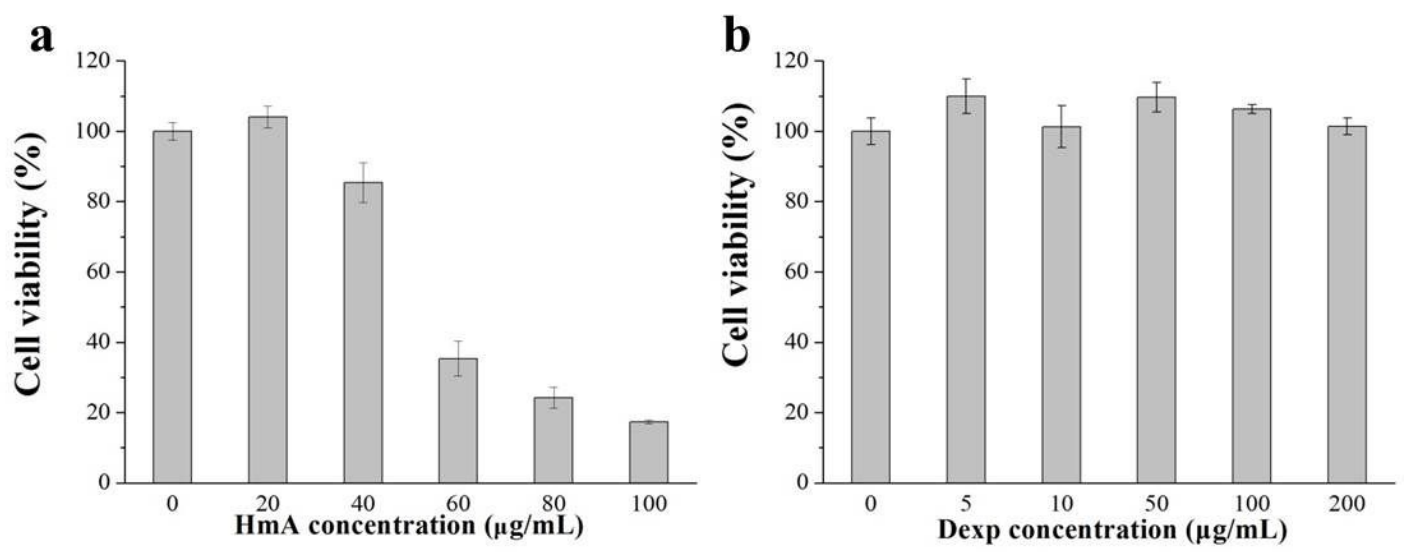

Figure S5. Cell viability of RAW264.7 cells incubated with different concentrations of (a) HmA and (b) Dexp for $24 \mathrm{~h}$. 
Table S1. Score obtained from eye irritation assessment of HmA (3.12 $\mathrm{mg} / \mathrm{mL}$ ) in rabbits

\begin{tabular}{lcl}
\hline & & Scores from \\
Ocular presentations & Scores & \\
& & assessment
\end{tabular}

A - Conjunctival edema (Chemosis)

Absent

0

Any swelling

Prominent swelling along with partial lid eversion

Swelling with half-closed lids

Swelling with totally closed lids

B - Redness in conjunctiva

Absent

Abnormal conjunctival injections

More diffuse and deeper hyperaemia, separate vessels can

not be seen easily

Diffuse and dense hyperaemia

C - Secretion 
Any abnormal secretion

Secretion leading to wet eye lashes closer to lids

D - Corneal opacity

Absent

Scattered or diffused areas - details of the iris discernible

Easy discernable, transparent areas, detail of the iris

slightly darkened

Opalescent areas, no details of the iris discernible, size of

the pupil barely discernible

Opaque cornea, iris not discernible

$\mathrm{E}-$ Iris involvement

Absent

Pronounced deep folds, congestion, deep swelling,

circumcorneal injection, the iris still reacts to light

No response, haemorrhage, marked destruction 



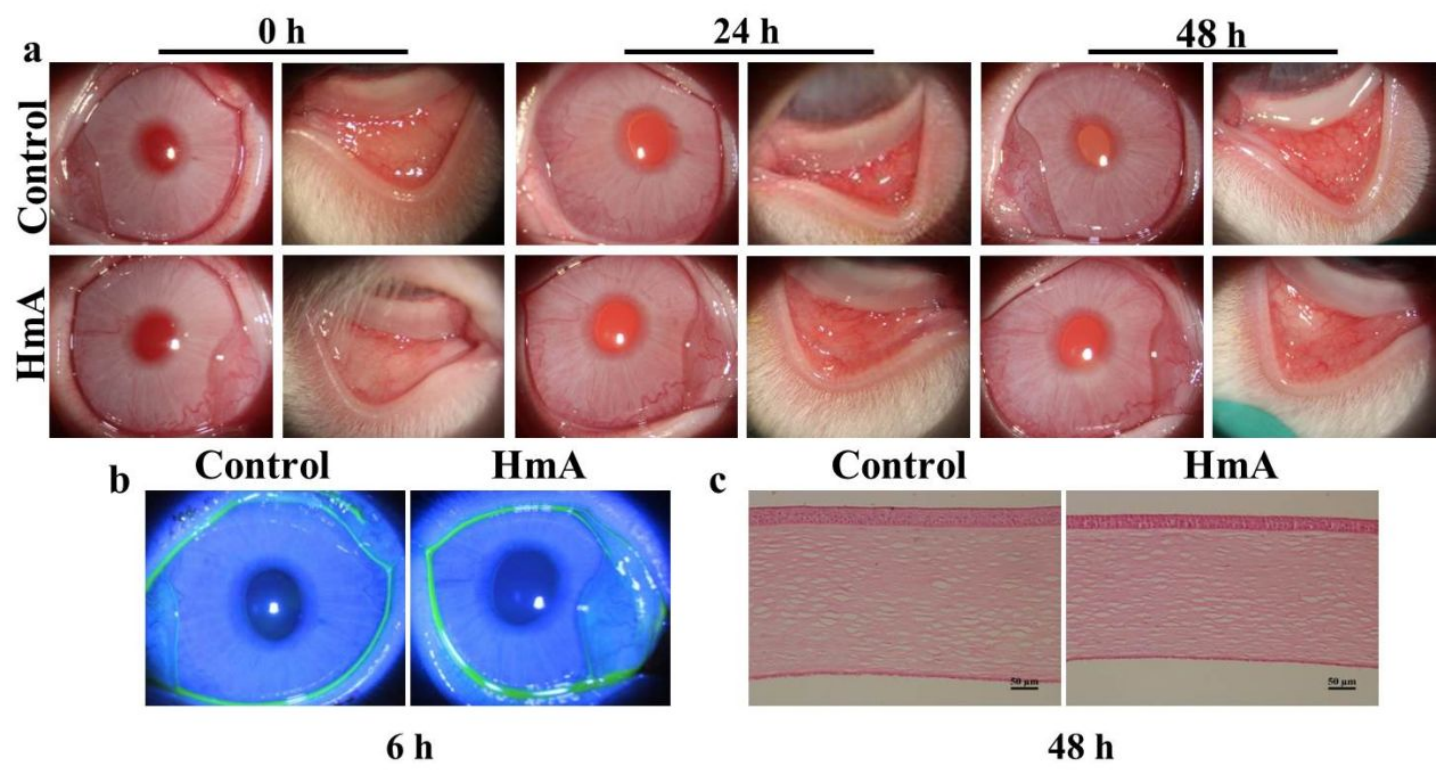

Figure S6. Ocular tolerance of HmA. (a) Images under slit lamp on different time after instillation. (b) Images under slit lamp with fluorescence sodium staining images after 6h instillation. (c) Histological cross-sections of cornea after $48 \mathrm{~h}$ instillation. 


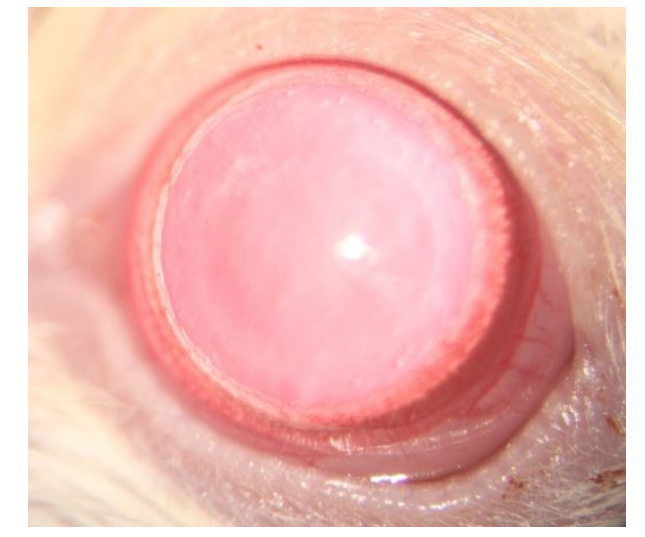

Figure S7. A picture of normal cornea. 


\section{Reference}

1. Bozdag, S.; Gumus, K.; Gumus, O.; Unlu, N., Formulation and in vitro evaluation of cysteamine hydrochloride viscous solutions for the treatment of corneal cystinosis. European Journal of Pharmaceutics and Biopharmaceutics 2008, 70 (1), 260-269. DOI: 10.1016/j.ejpb.2008.04.010. 